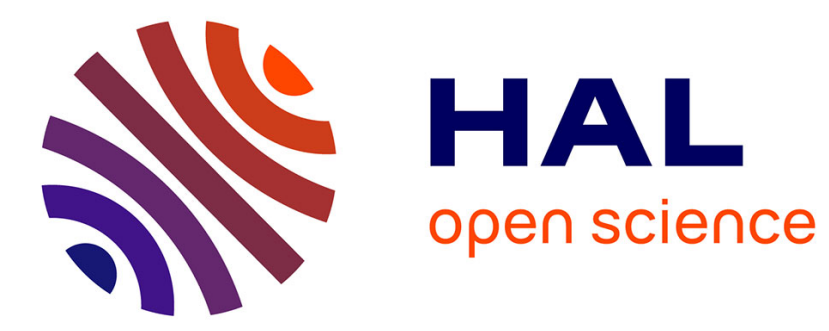

\title{
Arrays of high aspect ratio magnetic microstructures for large trapping throughput in lab-on-chip systems
}

\author{
Samir Mekkaoui, Damien Le Roy, Marie-Charlotte Audry, Joël Lachambre, \\ Véronique Dupuis, Jérôme Desgouttes, Anne-Laure Deman
}

\section{- To cite this version:}

Samir Mekkaoui, Damien Le Roy, Marie-Charlotte Audry, Joël Lachambre, Véronique Dupuis, et al.. Arrays of high aspect ratio magnetic microstructures for large trapping throughput in lab-onchip systems. Microfluidics and Nanofluidics, 2018, 22 (10), pp.119. 10.1007/s10404-018-2141-6 . hal-02326961

\section{HAL Id: hal-02326961 \\ https://hal.science/hal-02326961}

Submitted on 22 Oct 2019

HAL is a multi-disciplinary open access archive for the deposit and dissemination of scientific research documents, whether they are published or not. The documents may come from teaching and research institutions in France or abroad, or from public or private research centers.
L'archive ouverte pluridisciplinaire HAL, est destinée au dépôt et à la diffusion de documents scientifiques de niveau recherche, publiés ou non, émanant des établissements d'enseignement et de recherche français ou étrangers, des laboratoires publics ou privés. 


\title{
Arrays of high aspect ratio magnetic microstructures for large trapping throughput in Lab-On-Chip systems
}

\author{
Samir Mekkaoui ${ }^{1}$, Damien Le Roy ${ }^{2}$, Marie-Charlotte Audry ${ }^{1}$, Joël Lachambre ${ }^{3}$, Véronique Dupuis ${ }^{2}$, Jérôme \\ Desgouttes $^{1}$, Anne-Laure Deman ${ }^{1}$ \\ ${ }^{1}$ Institut des Nanotechnologies de Lyon INL-UMR5270, CNRS, Université Lyon 1, Villeurbanne F-69622, France \\ ${ }^{2}$ Institut Lumière Matière ILM-UMR 5306, CNRS, Université Lyon 1, Villeurbanne F-69622, France Laboratoire \\ ${ }^{3}$ Laboratoire Matériaux, Ingénierie et Sciences (MATEIS), INSA-Lyon, CNRS, UMR 5510, 20 Av. Albert \\ Einstein, 69621 Villeurbanne, France
}

Corresponding author: Samir Mekkaoui, samir.mekkaoui@univ-lyon1.fr ORCID: 0000-0002-9898-4495

\begin{abstract}
Here we report a novel technology that requires simple fabrication process, to obtain highly efficient magnetic micro-traps. Developed micro-traps consist in chains of iron particles diluted in PolyDiMethylSiloxane (PDMS). X-ray tomography was used to analyze the microstructure of particle ordering in the PDMS membrane and revealed the predominance of chain-like agglomerates. Largest formed traps, with diameter ranging from 4 to $11 \mu \mathrm{m}$ are found to be the most efficient. The self-organized trap arrays are characterized by a density of 1300 magnetic microtraps $/ \mathrm{mm}^{2}$, with an average nearest neighbour distance, center-to-center, of $21 \mu \mathrm{m}$. Implemented in a microfluidic channel operating at a flow rate of $3 \mathrm{~mL} / \mathrm{h}$ - a fluid flow of 8,3 $\mathrm{mm} / \mathrm{s}$ - we measured trapping throughputs up to 7100 beads/min with an average distribution of 750 beads $/ \mathrm{mm}^{2}$. At fluid velocity up to $9,7 \mathrm{~mm} / \mathrm{s}$ a trapping efficiency of $99,99 \%$ were measured. This novel technology allows to trap thousands of beads with throughputs that permit to compete with hydrodynamic trapping functions, while requiring simple fabrication process, and handling.
\end{abstract}

\section{Introduction}

Lab on a chip (LOC) technology brings numerous benefits for biomedical and environmental applications. LOC significantly reduces the amount of biological sample and reagent, the cost and the time of analysis, and it enables individual cell investigations. One of the most appealing potential of LOC is certainly the development of delocalized analysis solutions, performed in laboratories. For biomedical purpose, LOC working principle involves the manipulation of cells or functionalized beads, generally seeking either high throughputs or precise positioning and manipulation of single objects. In this scope, various solutions, including hydrodynamic, optical, dielectrophoretic, magnetic or acoustic actuations were developed. B. Cetin et al. (Cetin et al. 2014) made an extensive comparison of these techniques based on their implementation, microfabrication and materials and their performances, in particular throughput, considering clinical and diagnostic applications. All techniques present advantages or drawbacks that are weighted differently according to the use. When high sorting throughputs are required, hydrodynamic, acoustic and magnetic approaches are the most competitive. Single cell analysis also represents a strong challenge and aroused great interest in the LOC community during the past ten years (Hosic et al. 2015; Narayanamurthy et al. 2017). Indeed, it permits to reveal individual cell characteristics, due to cell-to-cell variation, cell cycle stages, or rare cell event and to access statistics about cells heterogeneity (Hosic et al. 2015; Yesilkoy et al. 2016; Narayanamurthy et al. 2017), hidden in global studies of large population. Similarly, manipulation of single microbeads has attracted a lot of interest recently (Kim et al. 2012; Tekin 
and Gijs 2013; Xu et al. 2013). Functionalized microbead arrays are indeed powerful tools to detect and quantify biological target (proteins, RNA, DNA, ...) or to trap labeled cells. The hydrodynamic contact-based approach has been widely developed to trap single microbead or cell (Chen et al. 2015; Yesilkoy et al. 2016; Delapierre et al. 2017; Narayanamurthy et al. 2017). Vertical traps such as microwells or lateral traps microfluidics such as U-shaped microstructures or pockets were precisely designed and used for this purpose. V. Narayanamurthy et al. have recently reviewed current achievements in microfluidics hydrodynamic trapping for single cell analysis. Even though this approach presents advantages, notable drawbacks remain. Hydrodynamic methods can require sophisticated fabrication processes owing to trap dimensions, target specific geometries, and in order to minimize fluidic flow disturbances inherent to trap footprints. Dedicated pumping systems and fluidic controls are often needed and add complexity in the device handling.

Magnetic forces can be promising for single objects trapping. This approach is based on magnetophoresis which refers to the motion of an object in a non-uniform magnetic field. Magnetophoresis is implemented in Lab On Chip (Dempsey et al. 2007; Li et al. 2011; Rasponi et al. 2011; Forbes and Forry 2012; Marchi et al. 2015), to perform the concentration, separation or trapping of magnetically labelled cells (Yu et al. 2011), deoxygenated red blood cells (Nam et al. 2013), or functionalized magnetic microbeads (Saliba et al. 2010). The magnetic method does not require cumbersome and specialized equipment which could hinder their handling by biological or medical laboratory. Indeed, magnetic forces can be generated by using either a simple external (bulk) permanent magnet (Mohamadi et al. 2015) or by a micrometer-sized magnetic structure nearby or within the microfluidic channel, named micro-magnets (Esmaeilsabzali et al. 2016). The latter approach permits to obtain larger magnetic field gradients, thus larger magnetic forces (Le Roy et al. 2016b) and to design target-size traps. Literature can be divided into two distinct kinds of micro magnets, made of hard magnetic materials (Dumas-Bouchiat et al. 2010; Arnold and Wang 2009), or soft magnetic materials (Dempsey et al. 2014; Deman et al. 2017; Jung et al. 2010). The first ones, permanently magnetized, work autonomously, but are limited to fixed force and necessitate costly and complex fabrication process, especially because its performances highly depend on the material microstructure (Zanini et al. 2011). The second ones need an external magnet in order to generate a magnetic field but offer the opportunity to easily tune the magnetic force during experiments and imply easier fabrication process. Usually, $\mathrm{Ni}$ or permalloy thin films are patterned by lithography to obtain micromagnets of typically $5-100 \mu \mathrm{m}$ in size (Chen et al. 2014; Jaiswal et al. 2017; Henighan et al. 2010). These processes still require several manufacturing steps, and face challenges related to the heterogeneous integration of magnetic materials with polymers, mainly PolyDiMethylSiloxane (PDMS), such as tedious alignment procedures (for locating the traps in the channels,) and tightness issues. Micromagnet arrays were reported to work as magnetic tweezers that enable precise manipulation of magnetic beads or cells (Henighan et al. 2010; Rampini et al. 2016), to traps rare cells (Chen et al. 2014) or for single cell analysis (Jaiswal et al. 2017).

We propose a novel technology to obtain efficient magnetic micro-trap arrays. The traps consist in high aspect ratio agglomerates of magnetic particles diluted in a polymer matrix. The magnetization and magnetic susceptibility are the two key parameters of soft micro-magnets as they determine the maximum reachable force. We have chosen iron to take benefit of its high magnetization. Through the formation of high aspect ratio agglomerates we induced an increased effective magnetic susceptibility (Khashan and Furlani 2014; Le Roy et al. 2016a). This novel technology, inspired by composite-polymer approach (Faivre et al. 2014; Zhou and Wang 2016; Royet et al. 2017), is low cost and requires simple fabrication process that breaks with standard microfabrication approaches. We obtained an array of 1300 magnetic micro- 
traps $/ \mathrm{mm}^{2}, 4$ to $11 \mu \mathrm{m}$ in diameter, spaced with an average nearest neighbour distance, centerto-center, of $21 \mu \mathrm{m}$. In this study, we characterized the composite structure using X-ray tomography and Atomic Force Microscopy (AFM). We characterized the trapping performances when implemented in microfluidic channel, with model superparamagnetic beads. Our findings show high efficiency with $99.99 \%$ of injected beads trapped at flow rates of 3 and $3.5 \mathrm{~mL} / \mathrm{h}$, respectively flow velocities of 8.3 and $9.7 \mathrm{~mm} / \mathrm{s}$. We measured throughputs up to 7100 trapped beads/min. Considering the achievable trap size and areal density, these materials could be used for single cell assays. The final part of this report is dedicated to this application, notably assessing the fraction of traps occupied by single objects.

\section{Microfabrication technology}

\subsection{Fabrication of the composite membrane integrating chains of particles}

The composite (I-PDMS) was obtained through the mixture of carbonyl iron microparticles (Fe-C dry powder, 0.5-7 $\mu \mathrm{m}$ diameter, 97\% Fe basis, Sigma-Aldrich) and PDMS elastomer (10/1 ratio of monomer and curing agent, respectively, Sylgard from Samaro) with concentration of $1 \mathrm{wt} \%$ and $5 \mathrm{wt} \%$. They were mixed in a mortar for around 4 minutes until obtaining a visually homogeneous material. Fig. 1 shows the fabrication steps. Composite membranes were patterned using $100 \mu \mathrm{m}$ thick molds made of Kapton film (Adicaz, France), bonded on silanized glass slice. Two sizes of molds were prepared: $10 \mathrm{~mm}$ x $10 \mathrm{~mm}$ and 10 $\mathrm{mm} \times 30 \mathrm{~mm}$. To create high aspect ratio magnetic agglomerates extended through the composite membrane thickness, a magnetic field was applied during the PDMS reticulation for 3 hours at $70^{\circ} \mathrm{C}$ (Fig. 1a-b). The magnetic field is created by a pair of permanent magnet of 10 $\mathrm{x} 11 \times 2.5 \mathrm{~cm}^{3}$, spaced of $6 \mathrm{~cm}$. The mold containing the composite was positioned onto one magnet, where a magnetic field of $150 \mathrm{mT}$ was measured using a Keithley Teslameter. The opposite magnet permits to significantly reduce the magnetic field gradient across the composite. At the position of the composite ( $1 \mathrm{~mm}$ from the magnet surface), the magnetic field gradient is of $0.25 \mathrm{~T} / \mathrm{m}$, as calculated using Comsol ${ }^{\circledR}$ simulation tools. After reticulation, the Kapton mold was then removed, leaving a $100 \mu \mathrm{m}$ thick patterned composite membrane (Fig. 1c). Pure PDMS (10/1 ratio of monomer and cross-linking agent) was poured on the composite, cured at $70^{\circ} \mathrm{C}$ for $3 \mathrm{~h}$, and peeled off from the glass slice to obtain a $2 \mathrm{~mm}$ thick PDMS/composite membrane (Fig. 1d-e).

\subsection{Device fabrication}

We patterned channels in pure PDMS matrix following a conventional soft-lithography process, using $50 \mu \mathrm{m}$ dry photoresist (Ethertec ${ }^{\circledR}$ ) (Fig. 1f-h). Channel dimension was $100 \mu \mathrm{m}$ thick (two layer of photoresist) and $1000 \mu \mathrm{m}$ width. We sealed the channel with the as-prepared magnetic membranes using $0_{2}$ plasma bonding (Fig. 1i). A schematic of the obtained microdevice is shown on Fig. 1j. 
a)

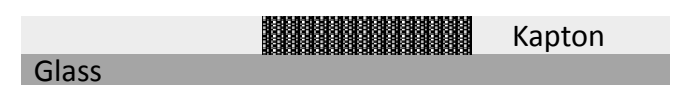

b)

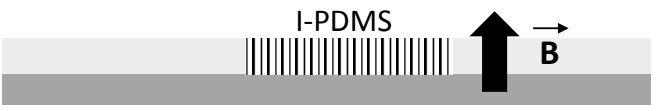

c)

d)

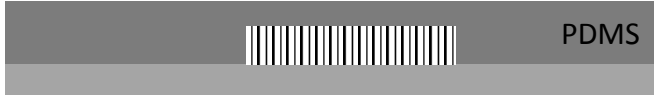

e)

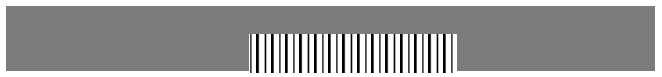

f)

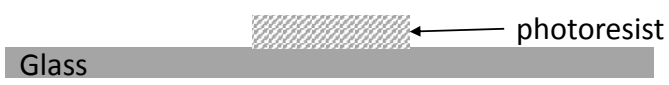

g)

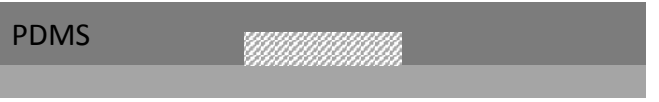

h)

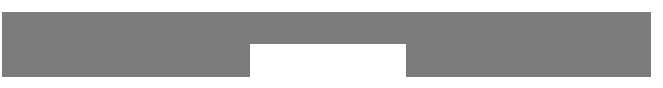

i)

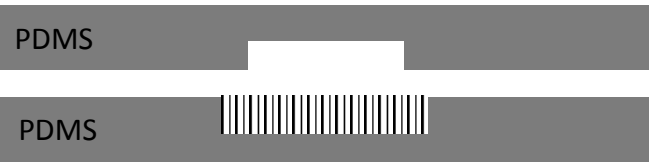

j)

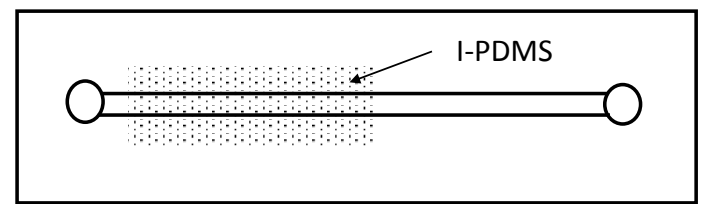

Fig. 1 Fabrication process. (a) A Kapton film is patterned on a glass slide and I-PDMS ( $1 \mathrm{wt} \%$ or $5 \mathrm{wt} \%)$ is poured in the pattern. A blade is used to remove the excess of composite (b) The I-PDMS is cured for $3 \mathrm{~h}$ at $70^{\circ} \mathrm{C}$ while being submitted to a vertical magnetic field of $\mathrm{B}=130 \mathrm{mT}$, in order to self-organize Fe-C particles in the PDMS matrix (c) The Kapton film is removed leaving only the self-organized composite membrane (d) PDMS is poured on the membrane, then cured for $3 \mathrm{~h}$ at $70^{\circ} \mathrm{C}$. (e) The PDMS/I-PDMS membrane is unmolded. (f) The microfluidic channel mold is obtained through photolithography g) PDMS is poured on the mold to replicate the mold and (h) unmolded (i) $\mathrm{O}_{2}$ plasma bonding is performed to seal the device comprising the microfluidic channel and the composite membrane. (j) is schematic of the device. Dots represent the location of the composite in the device.

\subsection{Material and experimental set-up}

The inner structure of the composite was characterized using X-ray tomography. These experiments were conducted on $1 \mathrm{wt} \%$ and $5 \mathrm{wt} \%$ I-PDMS concentration using "EasyTom Nano" $\mu \mathrm{CT}$ tomograph from "RX Solutions" company. The X-ray source is a LaB6 cathode with a diamond window to allow higher flux $(20 \mu \mathrm{A})$ and the used focal spot is $0.25 \mu \mathrm{m}$ width knowing that scans are done with a $0.3 \mu \mathrm{m}$ voxel size. As $\mathrm{Fe}-\mathrm{C}$ is quite absorbent, a tension of $90 \mathrm{kV}$ is applied. A CCD detector with a matrix of $2000 \times 1312$ pixel was used to take projections over 1400 angular positions. After reconstruction, a volume of $1800 \times 1100 \times 240$ vx, i.e. $540 \times 330 \times 72 \mu \mathrm{m}^{3}$, is obtained. Post treatment are done using ImageJ software in order to get a 3D picture of the sample, but also to extract qualitative results as spatial organization of the particles and quantitative results as effective volume fraction or aspect ratio adopted by those soft structures. Composite topographies were recorded with AFM (Asylum Research MFP-3D) in the tapping mode with silicon probes of nominal radius of curvature $10 \mathrm{~nm}$ and nominal spring constant $42 \mathrm{~N} / \mathrm{m}$.

Concerning trapping function evaluation in microchannel, superparamagnetic microbeads (12 $\mu \mathrm{m}$ average diameter, consisting of magnetite nano-inclusions in a polystyrene matrix, Kisker $(\mathbb{B})$ were suspended in a filtered Phosphate Buffered Saline (PBS) (Invitrogen) with a 
concentration of $10^{3}$ beads $/ \mu 1$. During the experiment, the microfluidic channel was positioned on a $\mathrm{NdFeB}\left(6 \times 3 \times 1.5 \mathrm{~cm}^{3}\right)$. It generated a magnetic field in the channel, i.e. at $2 \mathrm{~mm}$ from the magnet surface, of $300 \mathrm{mT}$ (measured using a Keithley Teslameter). Characterization of beads trapping and release was realized using a microscope (Olympus BX51M) and recorded using a camera (Moticam2000, Motic). Obtained images were analyzed using ImageJ ${ }^{\circledR}$ software.

\section{Results and discussion}

\subsection{Composite microstructuration}

Under an external magnetic field, carbonyl iron particles can be seen as an ensemble of aligned magnetic dipoles. Before complete PDMS reticulation, the particles are allowed to move within the polymer, and their motion is governed by dipolar interactions and gravity. Adjacent particles are attracted along the flux lines and repelled in the orthogonal directions. These directional interactions lead to the formation of high aspect ratio agglomerates along the flux lines, homogeneously distributed within the matrix. Fig. 2 displays reconstructed 3D profiles from $\mathrm{X}$-ray tomography performed on a volume of $540 \times 330 \times 72 \mu \mathrm{m}^{3}$. The deduced Fe-C particles size, ranging from 0.5 to $6 \mu \mathrm{m}$, is in good agreement with our SEM (Scanning Electron Microscopy) observations. For both concentrations, $1 \mathrm{wt} \%$ and $5 \mathrm{wt} \%$, the tomography images revealed two types of organizations: expected chain-like agglomerates (CA), but also isotropic agglomerates (IA). CA only represent $42 \%$ of all agglomerates in both $1 \mathrm{wt} \%$ and $5 \mathrm{wt} \%$ composites but contain $92 \%$ and $96 \%$ of the total amount of Fe-C particles, respectively. IA sizes are found to be less than $6 \mu \mathrm{m}$, and mostly localized at the bottom of the membrane (fig 2.b). Since the magnetic field gradient during cross-linking is rather small, $0.25 \mathrm{~T} / \mathrm{m}$ (calculated using Comsol ${ }^{\circledR}$ simulation tools), we believe that this concentration of IA at the bottom mainly originates from the gravitational force.

a) $\quad 1 \mathrm{wt} \%$

${ }_{y}^{z} x^{2}$<smiles>[3H][3H]</smiles>
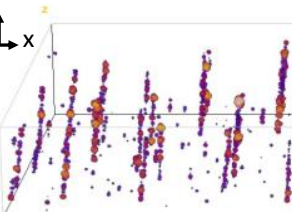

b)

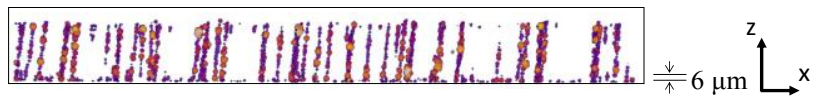

c)
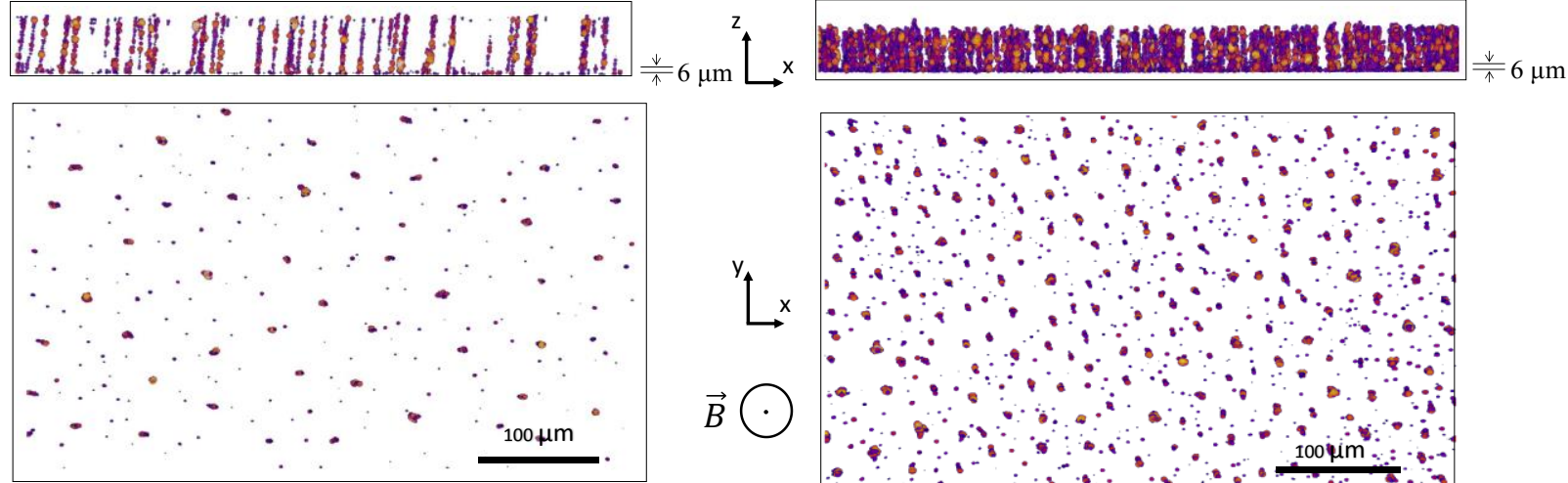

Fig. 2 (a) X-ray tomography reconstruction and projections on (b) XZ plane, bottom of images (6 $\mu \mathrm{m}$ high) cropped in order to remove IA is shown, and (c) XY plane

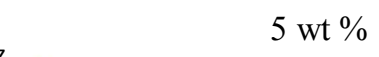

${ }_{y} \rightarrow x$
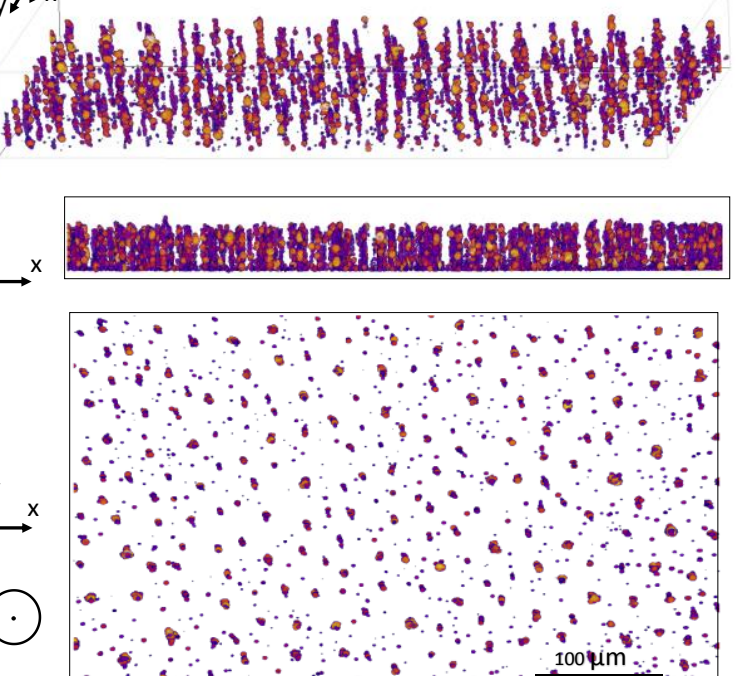
When implemented into microfluidic devices, these agglomerates locally generate magnetic

We found that $1 \mathrm{wt} \%$ and $5 \mathrm{wt} \%$ composites are characterized by densities of around 1500 traps $/ \mathrm{mm}$ and 5000 traps $/ \mathrm{mm}$, respectively, without distinction between CA and IA. Fig 4 . shows that the distribution of trap diameters exhibits two maxima, centered at 2 and $7 \mu \mathrm{m}$ in both composite concentrations. In the following discussion, for the sake of simplicity, we will refer to two populations of traps, "small traps" and "large traps", corresponding both peaks of the size distribution, the discriminating diameter being $4 \mu \mathrm{m}$. Small traps are predominant as they represent almost $81 \%$ of traps at $1 \mathrm{wt} \%$ composite and $74 \%$ of traps at $5 \mathrm{wt} \%$. To relate the CA and IA structures to the trap size, we reconstructed a new batch of tomography images after cropping a $6 \mu \mathrm{m}$-thick superficial layer in the ZX plane of all images, so that to remove IA (Fig. $2 b)$. The resulting cropped images permit to specifically characterize CA structures. Fig. 4a reports the diameter distribution from IA and CA structures for $1 \mathrm{wt} \%$ and $5 \mathrm{wt} \%$ composite membranes. At $5 \mathrm{wt} \%$ the proportion of thicker CA structures is slightly increased, like it has been already observed by other groups (Ghosh and Puri 2013; Günther et al. 2011). Most of IA structures, 94 to $97 \%$ for each composite, gave rise to "small traps" as their diameter is inferior to $4 \mu \mathrm{m}$. Fig. $4 \mathrm{~b}$ shows the proportion of traps originating form IA and CA structures based on the discriminating diameter of $4 \mu \mathrm{m}$ for small and large traps. $90 \%$ of large traps observed on the membrane surface originate from CA structures. 


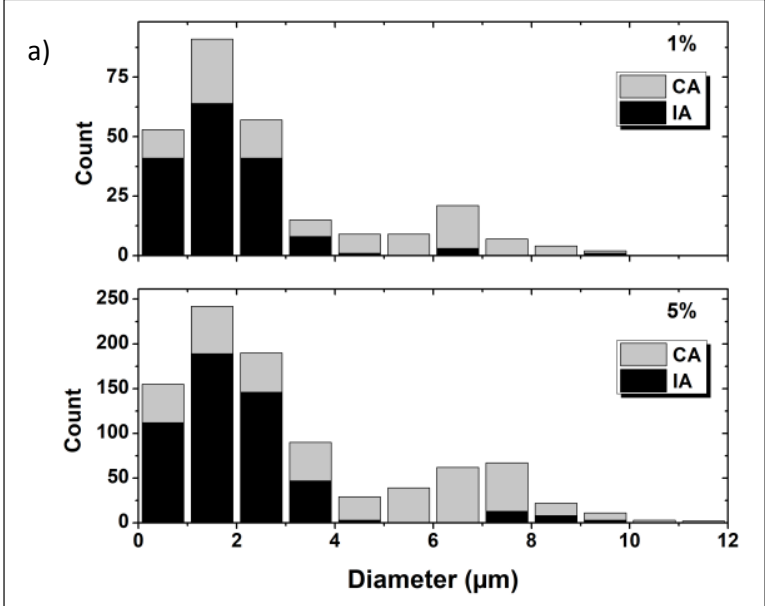

b)

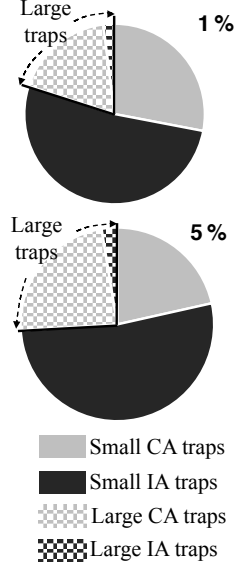

Fig. 4 (a) Distribution of traps diameter of $1 \mathrm{wt} \%$ membrane (top) and $5 \mathrm{wt} \%$ membrane (bottom), in black the IA proportion and in grey the CA proportion. (b) Pie charts showing the trap size distribution depending on their structure, in black the proportion of IA and in grey the proportion of CA. The checkboards pattern represents all traps exhibiting a diameter $\mathrm{D}>4 \mu \mathrm{m}$ (large traps).

Using optical microscopy at a magnification of $\mathrm{x} 100$, which possesses a lower resolution compared to X-ray tomography, only larger traps are visible. Microfluidic observations realized with an optical microscope have shown that trapping only occurred on visible traps, i.e. larger traps, that originate for $90 \%$ of them from CA structure. This is consistent with the fact that this structure is expected to be more efficient, taking benefit of it high aspect ratio which favors the concentration of magnetic field lines (Khashan and Furlani 2014). Considering that (i) the density of traps is of first importance in the trapping capacity of the device, and (ii) larger traps are the efficient ones, we focused our attention on the characterization of the population of large traps, 4 to $11 \mu \mathrm{m}$ in diameter, of $5 \mathrm{wt} \%$ sample. Their density reaches $1300 \mathrm{traps} / \mathrm{mm}^{2}$ with nearest neighbour distances center-to-center ranging from 17 to $27 \mu \mathrm{m}$ as reported on Fig. 5 . This trapping array specification suits for our beads models ( $12 \mu \mathrm{m}$ diameter) but also for larger target such as WBCs, or cells.

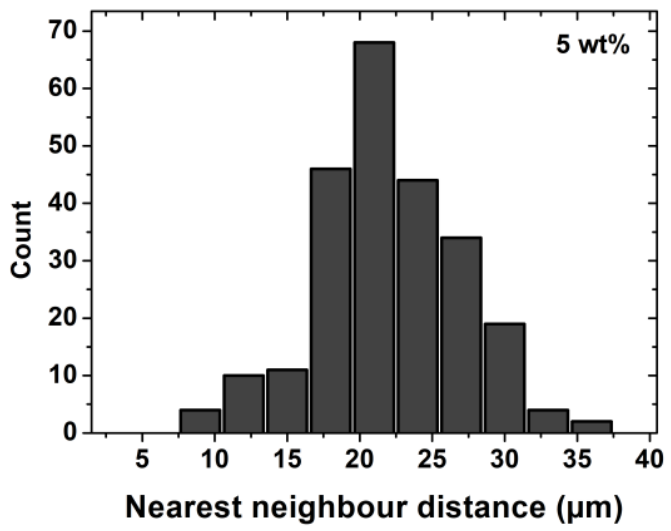

Fig. 5 Nearest neighbour distances, center-to-center, for large traps (diameter $>4 \mu \mathrm{m}$ ) of $5 \mathrm{wt} \%$ composite

\subsection{Trapping characterization in microfluidic channel}

In our experimental set-up, the microfluidic device is positioned on a $\mathrm{NdFeB}$ magnet that generates a $300 \mathrm{mT}$ magnetic field in the channel, i.e. at $2 \mathrm{~mm}$ from the magnet surface. From 
COMSOL ${ }^{\circledR}$ simulations, we expect a $18.2 \mathrm{~T} / \mathrm{m}$ magnetic field gradient originating from the magnet underneath the device. In order to estimate the effect of the magnet alone, we first carried out a series of experiments without magnetic composite. We observed an average of only $3 \mathrm{immobilized} \mathrm{beads} \mathrm{in} \mathrm{frame} \mathrm{at} 500 \mu \mathrm{L} / \mathrm{h}$ and none at $1 \mathrm{~mL} / \mathrm{h}$, on pure PDMS floor. This reference series of experiment ensures that in the following study, the trapping can be attributed to the composite membrane.

The $5 \mathrm{wt} \%$ membrane was then implemented into microfluidic devices, constituting the bottom side of a microfluidic channel (Fig. 1j). The trapping area was $1 \mathrm{~mm}$ large (channel width) and $1 \mathrm{~cm}$ long. Superparamagnetic beads were injected in the device with flow rate ranging between 1 to $3.5 \mathrm{~mL} / \mathrm{h}$, corresponding to flow velocities ranging between 2.8 to $9.7 \mathrm{~mm} / \mathrm{s}$. For flow rates below $3 \mathrm{~mL} / \mathrm{h}$, all beads were trapped (100\% trapping efficiency), individually or in clusters, during the gradual filling of the trapping area. The supplemental bead injection optimized the filling of the traps but also favored the formation of bead clusters.

For flow rate of $3 \mathrm{~mL} / \mathrm{h}$, few un-trapped beads were observed during the progressive filling of traps. We therefore studied the trapping characteristics of the device at the optimum flow rates of 3 and $3.5 \mathrm{~mL} / \mathrm{h}$. We timed the experiments until beads reached the end of the trapping area and then we stopped the flow. Fig. 6 a) reports a microscopic image of the traps in the channel before and after trapping of the beads once the flow was stopped. During experiments untrapped beads were counted in order to determine trapping efficiency. Table 1 shows the trapping efficiency, throughput, density of trapped beads, and ratio of traps occupied by one single beads, at 3 and $3.5 \mathrm{~mL} / \mathrm{h}$.
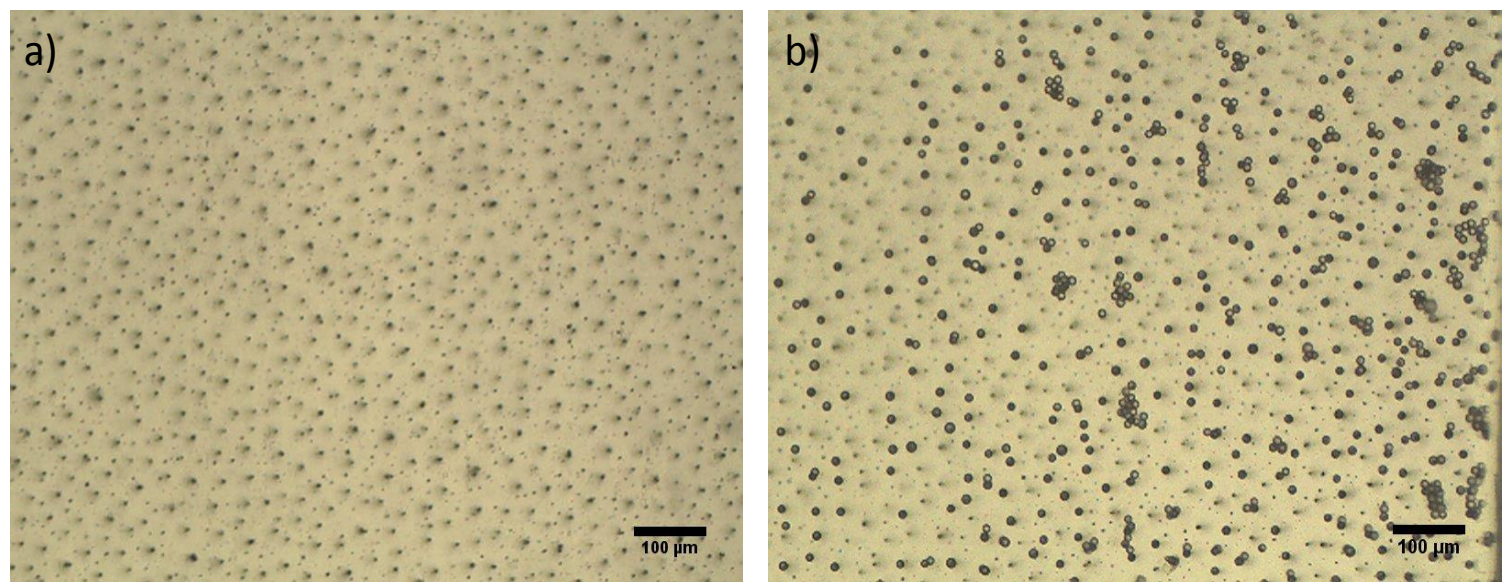

Fig. 6 Microscopic images of the trapping area (a) before and (b) after capture of the beads at a flow velocity of $9.8 \mathrm{~mm} / \mathrm{s}(3.5 \mathrm{~mL} / \mathrm{h})$.

The trapping efficiency of the device was found to be very high, in the order of $99.99 \%$. Experiments lasted $1 \mathrm{~min}$ and few seconds, and 7950 beads $\left(750\right.$ beads $\left./ \mathrm{mm}^{2}\right)$ and 5646 beads $\left(646\right.$ beads $\left./ \mathrm{mm}^{2}\right)$ were trapped at respectively 3 and $3.5 \mathrm{~mL} / \mathrm{h}$. Average throughput of 7100 trapped beads/min and 4733 trapped beads/min were calculated. These performances compete with the one reported for hydrodynamic approaches (Narayanamurthy et al. 2017). 
Table 1 Composite membrane trapping characteristics: throughput, trapping efficiency, number and density of trapped beads, ratio of traps occupied with a single bead, at flow rate of 3 and $3.5 \mathrm{~mL} / \mathrm{h}$, which correspond to flow velocities of 8.3 and $9.7 \mathrm{~mm} / \mathrm{s}$.

\begin{tabular}{ccccccc}
\hline \hline $\begin{array}{l}\text { Flow rate } \\
\mathrm{mL} / \mathrm{h}\end{array}$ & $\begin{array}{c}\text { Velocity } \\
\mathrm{mm} / \mathrm{s}\end{array}$ & $\begin{array}{c}\text { Throughput } \\
\text { beads/min }\end{array}$ & $\begin{array}{c}\text { Trapping } \\
\text { efficiency }\end{array}$ & $\begin{array}{c}\text { Number of trapped } \\
\text { beads }\end{array}$ & $\begin{array}{c}\text { Density } \\
\text { Beads/mm }\end{array}$ & $\begin{array}{c}\text { Traps with } \\
\text { single bead }\end{array}$ \\
\hline 3 & 8.3 & $7100+/-500$ & $99.99 \%$ & $7951+/-562$ & $750+/-57$ & $28 \%$ \\
\hline 3.5 & 9.7 & $4733+/-338$ & $99.99 \%$ & $5646+/-413$ & $656+/-41$ & $46.7 \%$
\end{tabular}

The trapping dynamics in the channel can be describe as follows. The first beads were captured on traps at the beginning of the trapping area and homogeneously across the channel width. The gradual filling of traps appeared heterogenous along the trapping area. Indeed, an average of $25 \%$ of the traps are occupied by single beads or clusters of beads in the total area, but $41 \%$ at its entrance and 5\% at its end. Fig. 7 reports the number of trapped beads at different positions in the channel. The dimensions of each observation window are $750 \mu \mathrm{m}$ long and $1000 \mu \mathrm{m}$ width, which corresponds to the channel width, they are numbered form N1 to N5, as represented on Fig. 7(a). For a flow velocity of $8.3 \mathrm{~mm} / \mathrm{s}$ (flow rate of $3 \mathrm{~mL} / \mathrm{h}$ ), the number of trapped beads decreases from 1085 (13.6\% of the total of trapped beads) in N1, to $591(7.4 \%)$ in N3 to $150(1.9 \%)$ in N5.

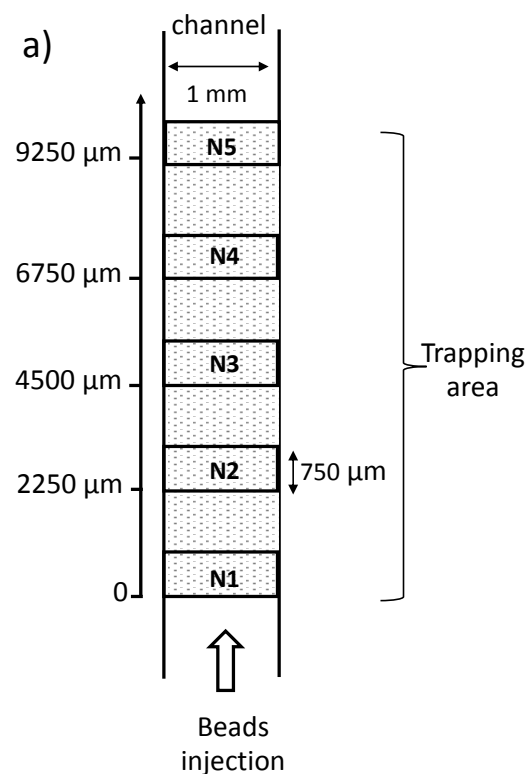

b)

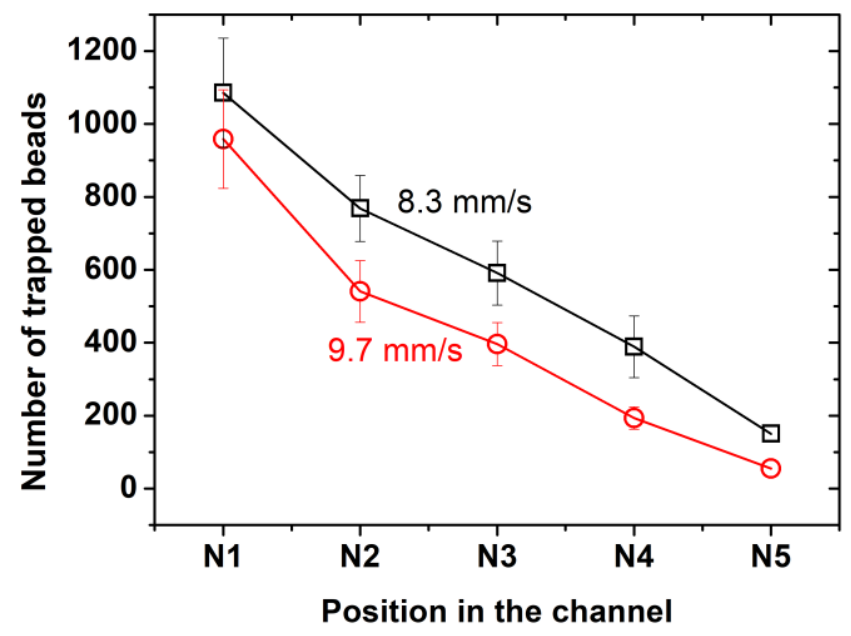

Fig. 7 (a) Schematic representation of observation windows, N1, N2, N3, N4 and N5, positioned at $0 \mu \mathrm{m}, 2250$ $\mu \mathrm{m}, 4500 \mu \mathrm{m}, 6750 \mu \mathrm{m}, 9250 \mu \mathrm{m}$ from the entrance of the trapping area, (b) number of trapped beads in the channel in each observation window.

Besides trapping dynamic, theses arrays of magnetic structures could be used to immobilize single objects, notably for assays on large populations of individual cells. In this view, we put the focus on the fraction of single beads occupying traps. With flow velocities of $8.3 \mathrm{~mm} / \mathrm{s}$ and $9.7 \mathrm{~mm} / \mathrm{s}, 28 \%$ and $46.7 \%$ of the traps were occupied by a single bead, the others by bead clusters, as reported on Table 1. The higher fraction of single beads at $9.7 \mathrm{~mm} / \mathrm{s}$ than at 8.3 $\mathrm{mm} / \mathrm{s}$ could be attributed to the relatively small cohesion of bead clusters, as expected from dipolar interactions between superparamagnetic beads, and that the formation of the clusters is 
essentially due to bead-trap interactions. Fig. 8 reports the ratio of traps occupied by a single bead. This ratio exceeds $50 \%$ in the middle of trapping area at $9.7 \mathrm{~mm} / \mathrm{s}$, whereas is remains lower than $32 \%$ à $8.3 \mathrm{~mm} / \mathrm{s}$.
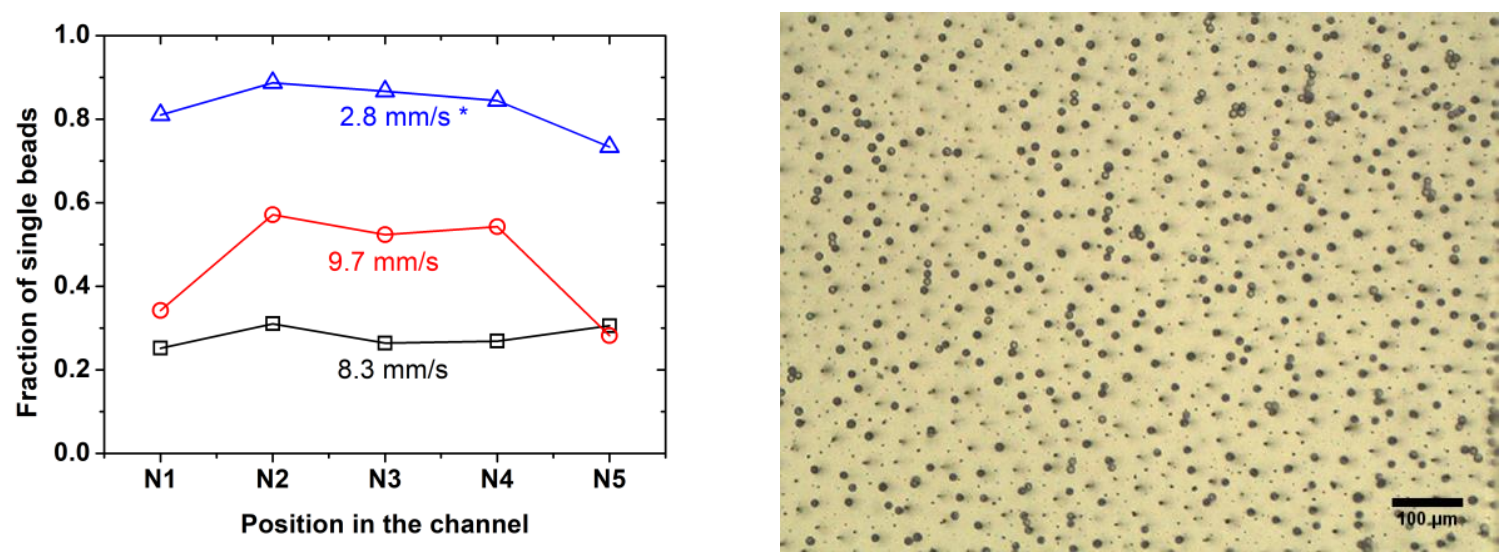

Fig. 8 (a) Fraction of filled traps with a single bead for flow velocities of $8.3 \mathrm{~mm} / \mathrm{s}, 9.7 \mathrm{~mm} / \mathrm{s}$ and $2.8 \mathrm{~mm} / \mathrm{s}$ followed by short accelerations. (b) Microscopic images of the trapping area after single bead trapping optimization (and $2.8 \mathrm{~mm} / \mathrm{s}$ followed by short accelerations)

The low cohesion of bead clusters permits to foresee different routes to increase the number of individual trapped beads in the device, notably by modulating the flow rate in time. To illustrate it, we injected beads in the device at lower flow rate, $1 \mathrm{~mL} / \mathrm{h}$ (flow velocity of $2.8 \mathrm{~mm} / \mathrm{s}$ ), in order to fill the entire trapping zone with beads and clusters. Then, to break beads clusters, we created short acceleration through pressure drop by moving capillary. Fig. 8 (b) reports a microscopic image of beads trapping in the device. One can observe the absence of large beads clusters and a larger proportion of single bead trapped. As reported on Fig. 8, the traps occupied by a single bead represent $73 \%$ to $88 \%$ of occupied traps. A better control of the short accelerations could permit to precisely monitor the coverage of the microbead array.

\section{Conclusion}

In this paper, we propose a novel technology to obtain efficient magnetic micro-traps. This novel technology, inspired by composite-polymer approach, is low cost and requires simple fabrication process that break with standard microfabrication processes. Developed microtraps consist of chains of Fe-C microparticles diluted in the PDMS. X-ray tomography experiments gave significantly better insights of microstructure engineering mechanisms for low $\mathrm{Fe}-\mathrm{C}$ concentration magnetic composites ( $1 \mathrm{wt} \%$ and $5 \mathrm{wt} \%$ ). The self-organization of Fe-C particles in a PDMS matrix leads to the creation of highly dense arrays of chain-like agglomerates in large proportion, together with sparse isotropic small agglomerates. Larger traps, originated for $90 \%$ of them from CA structure, were identified as the most efficient ones. Their density reached 1300 magnetic microtraps $/ \mathrm{mm}^{2}$, with diameter from 4 to $11 \mu \mathrm{m}$, and an average nearest neighbour distance of $21 \mu \mathrm{m}$. They were implemented in a microfluidic channel. We assessed the trapping efficiency and throughput of the device and studied the trapping dynamics. We obtained trapping efficiency of $99,99 \%$ at flow velocities of 8.3 to $9.7 \mathrm{~mm} / \mathrm{s}$. At $8.3 \mathrm{~mm} / \mathrm{s}$, throughputs up to 7100 trapped beads/min were measured. At $9,7 \mathrm{~mm} / \mathrm{s}$ the ratio on single bead traps was improved. This novel technology allows to trap thousands of beads with throughputs that, to the best of our knowledge, were never reported using magnetic forces and that permit to compete with hydrodynamic trapping functions, while requiring simple fabrication process, and handling. 


\section{Acknowledgements}

We wish to acknowledge support for the project from Ecole doctorale EEA and regional AURA financial support, Nanolyon technological platform, MATEIS lab for X-ray tomography analyses, and A. Piednoir for AFM characterization at ILM.

\section{References}

Arnold DP, Wang N (2009) Permanent magnets for MEMS. J Microelectromechanical Syst $18: 1255-1266$

Cetin B, Özer MB, Solmaz ME (2014) Microfluidic bio-particle manipulation for biotechnology. Biochem Eng J 92:63-82

Chen H, Sun J, Wolvetang E, Cooper-White J (2015) High-throughput, deterministic single cell trapping and long-term clonal cell culture in microfluidic devices. Lab Chip 15:10721083

Chen P, Huang Y-Y, Hoshino K, Zhang X (2014) Multiscale immunomagnetic enrichment of circulating tumor cells: from tubes to microchips. Lab Chip 14:446-458

Delapierre F-D, Mottet G, Taniga V, et al (2017) High throughput micropatterning of interspersed cell arrays using capillary assembly. Biofabrication 9:015015

Deman A-L, Chateaux J-F, Dhungana D, et al (2017) Anisotropic composite polymer for high magnetic forces in microfluidic systems. Microfluid. Nanofluidics

Dempsey NM, Le Roy D, Marelli-Mathevon H, et al (2014) Micro-magnetic imprinting of high field gradient magnetic flux sources. Appl Phys Lett 104:262401

Dempsey NM, Walther A, May F, et al (2007) High performance hard magnetic NdFeB thick films for integration into micro-electro-mechanical systems. Appl Phys Lett 90:092509

Dumas-Bouchiat F, Zanini L-F, Kustov M, et al (2010) Thermomagnetically patterned micromagnets. Appl Phys Lett 96:102511

Esmaeilsabzali H, Beischlag TV, Cox ME, et al (2016) An integrated microfluidic chip for immunomagnetic detection and isolation of rare prostate cancer cells from blood. Biomed Microdevices 18:22

Faivre M, Gelszinnis R, Degouttes J, et al (2014) Magnetophoretic manipulation in microsystem using carbonyl iron-polydimethylsiloxane microstructures. Biomicrofluidics $8: 054103$

Forbes TP, Forry SP (2012) Microfluidic magnetophoretic separations of immunomagnetically labeled rare mammalian cells. Lab Chip 12:1471-1479

Ghosh S, Puri IK (2013) Soft polymer magnetic nanocomposites: microstructure patterning by magnetophoretic transport and self-assembly. Soft Matter 9:2024-2029 
Günther D, Borin DY, Günther S, Odenbach S (2011) X-ray micro-tomographic characterization of field-structured magnetorheological elastomers. Smart Mater Struct 21:015005

Henighan T, Chen A, Vieira G, et al (2010) Manipulation of magnetically labeled and unlabeled cells with mobile magnetic traps. Biophys J 98:412-417

Hosic S, Murthy SK, Koppes AN (2015) Microfluidic sample preparation for single cell analysis. Anal Chem 88:354-380

Jaiswal D, Rad AT, Nieh M-P, et al (2017) Micromagnetic cancer cell immobilization and release for real-time single cell analysis. J Magn Mater 427:7-13

Jung Y, Choi Y, Han K-H, Frazier AB (2010) Six-stage cascade paramagnetic mode magnetophoretic separation system for human blood samples. Biomed Microdevices 12:637645

Khashan SA, Furlani EP (2014) Scalability analysis of magnetic bead separation in a microchannel with an array of soft magnetic elements in a uniform magnetic field. Sep Purif Technol 125:311-318

Kim H, Lee S, Kim J (2012) Hydrodynamic trap-and-release of single particles using dualfunction elastomeric valves: design, fabrication, and characterization. Microfluid Nanofluidics $13: 835-844$

Le Roy D, Dhungana D, Ourry L, et al (2016a) Anisotropic ferromagnetic polymer: A first step for their implementation in microfluidic systems. AIP Adv 6:056604

Le Roy D, Shaw G, Haettel R, et al (2016b) Fabrication and characterization of polymer membranes with integrated arrays of high performance micro-magnets. Mater Today Commun 6:50-55

Li J, Zhang M, Wang L, et al (2011) Design and fabrication of microfluidic mixer from carbonyl iron-PDMS composite membrane. Microfluid Nanofluidics 10:919-925

Marchi S, Casu A, Bertora F, et al (2015) Highly magneto-responsive elastomeric films created by a two-step fabrication process. ACS Appl Mater Interfaces 7:19112-19118

Mohamadi RM, Besant JD, Mepham A, et al (2015) Nanoparticle-Mediated Binning and Profiling of Heterogeneous Circulating Tumor Cell Subpopulations. Angew Chem 127:141145

Nam J, Huang H, Lim H, et al (2013) Magnetic separation of malaria-infected red blood cells in various developmental stages. Anal Chem 85:7316-7323

Narayanamurthy V, Nagarajan S, Samsuri F, Sridhar T (2017) Microfluidic hydrodynamic trapping for single cell analysis: mechanisms, methods and applications. Anal Methods 9:3751-3772

Rampini S, Li P, Lee G (2016) Micromagnet arrays enable precise manipulation of individual biological analyte-superparamagnetic bead complexes for separation and sensing. Lab Chip $16: 3645-3663$ 
Rasponi M, Piraino F, Sadr N, et al (2011) Reliable magnetic reversible assembly of complex microfluidic devices: fabrication, characterization, and biological validation. Microfluid Nanofluidics 10:1097-1107

Royet D, Hériveaux Y, Marchalot J, et al (2017) Using injection molding and reversible bonding for easy fabrication of magnetic cell trapping and sorting devices. J Magn Mater 427:306-313

Saliba A-E, Saias L, Psychari E, et al (2010) Microfluidic sorting and multimodal typing of cancer cells in self-assembled magnetic arrays. Proc Natl Acad Sci 107:14524-14529

Tekin HC, Gijs MA (2013) Ultrasensitive protein detection: a case for microfluidic magnetic bead-based assays. Lab Chip 13:4711-4739

Xu X, Li Z, Kotagiri N, et al (2013) Microfluidic microsphere-trap arrays for simultaneous detection of multiple targets. International Society for Optics and Photonics, p 86151E

Yesilkoy F, Ueno R, Desbiolles B, et al (2016) Highly efficient and gentle trapping of single cells in large microfluidic arrays for time-lapse experiments. Biomicrofluidics 10:014120

Yu X, Feng X, Hu J, et al (2011) Controlling the magnetic field distribution on the micrometer scale and generation of magnetic bead patterns for microfluidic applications. Langmuir 27:5147-5156

Zanini L-F, Dempsey NM, Givord D, et al (2011) Autonomous micro-magnet based systems for highly efficient magnetic separation. Appl Phys Lett 99:232504

Zhou R, Wang C (2016) Microfluidic separation of magnetic particles with soft magnetic microstructures. Microfluid Nanofluidics 20:48 